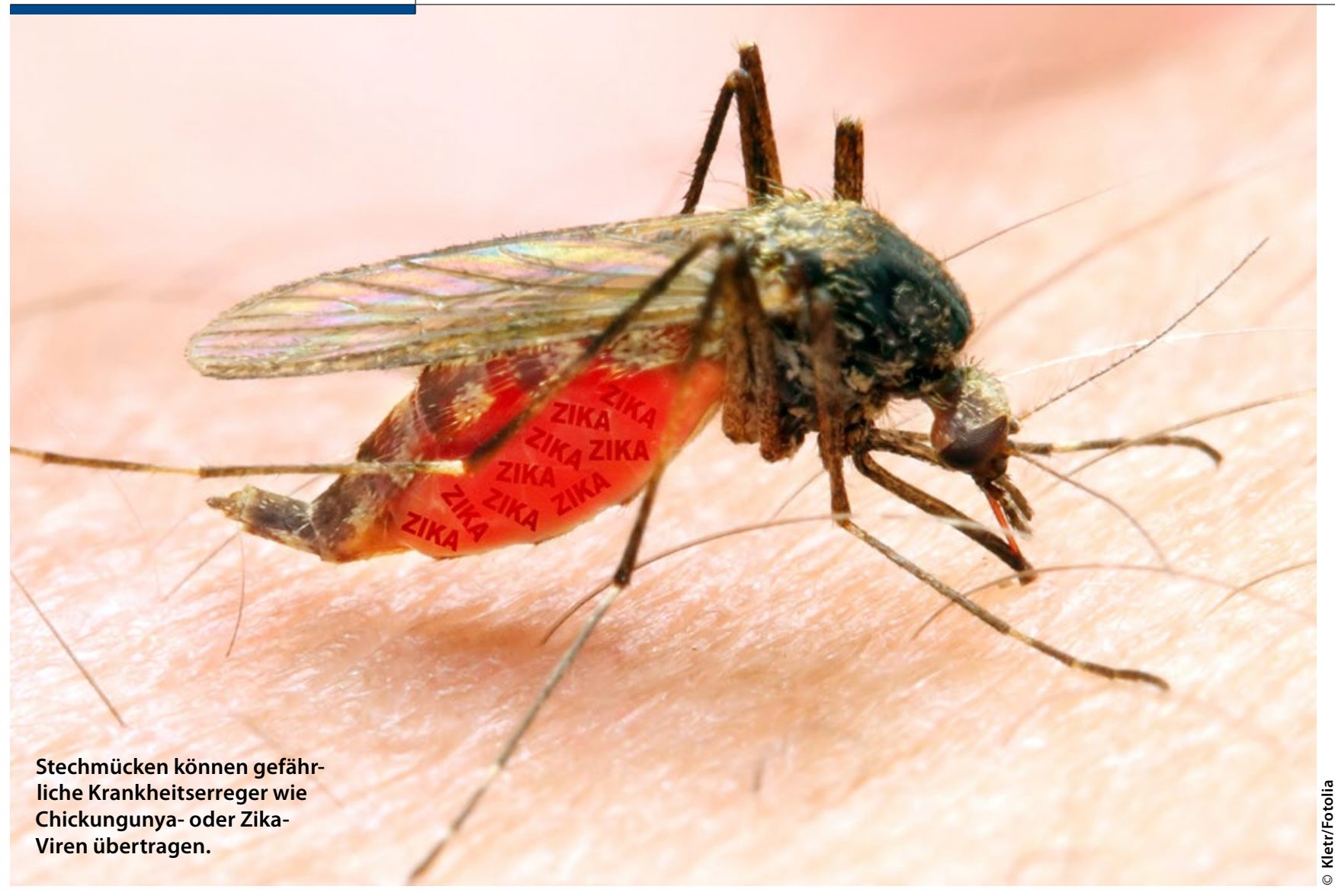

Ein vielschichtiges Thema

\title{
Rheuma und Infektionen
}

Infektionen können einerseits rheumatische Erkrankungen auslösen. Andererseits sind sie auch eine gefürchtete Komplikation unter einer antirheumatischen Therapie, insbesondere mit Biologika. Auch im perioperativen Kontext spielen sie eine wichtige Rolle, etwa wenn Bakterien zu Infektionen von Gelenkendoprothesen führen.

$\mathrm{B}$ ei infektiösen Auslösern von rheumatischen Erkrankungen wird zumindest in der Laienpresse meist an die Borreliose als Trigger einer Fibromyalgie gedacht. Eine aktuelle Studie konnte diesen Zusammenhang anhand der Klinik (Erythema migrans plus serologischer Befund) nicht bestätigen. Nach gut 15 Jahren fand sich nur bei einem der 100 Studienteilnehmer eine Fibromyalgie. Sie war demnach nicht häufiger als in der Allgemeinbevölkerung [1]. Die Lyme-Arthritis ist dagegen laut Prof. Christian Kneitz, Chefarzt der Klinik für Innere Medizin II, Rheumatologie/Klinische Immunologie, Geriatrie des Klinikums Südstadt, Rostock, zwar im Vergleich zum Erythe- ma migrans relativ selten, aufgrund der hohen Infektionsrate mit Borrellia burgdorferi jedoch in der Praxis eine relevante Manifestation der Infektion.

Wie Kneitz beim Rheuma-Update 2016 berichtete, scheinen bakterielle Infekte als Auslöser einer rheumatoiden Arthritis (RA) aber seltener zu sein als oft gedacht. Zumindest fand eine aktuelle populationsbasierte Fall-Kontroll-Studie keine signifikante Assoziation von Sinusitis, Tonsillitis, Zahnfleischinfektionen oder Pneumonien mit dem Auftreten einer RA. Für gastrointestinale, urogenitale und genitale Infektionen fand sich sogar ein erniedrigtes RA-Risiko [2]. Kneitz zufolge wird eine protektive Veränderung des Mikrobioms diskutiert. 
Differenzialdiagnose arthritogene Arboviren Das arthritogene, von Stechmücken übertragene Chickungunya-Virus aus der Familie der Arboviren, scheint dagegen aufgrund seiner weiten Verbreitung in der südlichen Hemisphäre durchaus ein relevanter Erreger von Arthritiden zu sein. Die Symptome gleichen sowohl in der Akutphase als auch im Langzeitverlauf denen einer RA mit Fieber (90\% der Fälle, eine Woche anhaltend), Myalgien (90\%, sieben bis zehn Tage) und Polyarthralgien/ Polyarthritis (95\%, Wochen bis Monate), die das Stammskelett aussparen und einer RA täuschend ähnlich sind; Hautreaktionen kommen ebenfalls häufig vor (40-50\%, eine Woche). Auch die immunologischen Phänomene gleichen sich. Ein Erregernachweis mittels PCR ist in den ersten Tagen möglich. Die Diagnose kann im weiteren Verlauf durch Antikörpernachweis bestätigt werden [3].

Nach einer US-amerikanischen Studie erfüllen acht von zehn Patienten die ACR-Kriterien für eine seronegative RA; das Muster der T-Zell-Populationen (mehr CD41- und CD81-positive T-Zellen) glich dem von RA-Patienten [4]. Somit ist die Chickungunya-Infektion laut Kneitz zumindest eine wichtige Differenzialdiagnose einer neu aufgetretenen RA. Der Rheumatologe riet zu einer Reiseanamnese und serologischen Diagnostik, wenn neben den Gelenkbeschwerden auch Fieber und ein Hautexanthem vorliegen.

Infizieren sich Patienten, die wegen einer rheumatischen Erkrankung mit Biologika behandelt werden, mit dem Chickungunya-Virus, ist ihr Risiko für einen schweren Verlauf nicht erhöht [5]. Rheuma-Kranke müssen also nicht auf Fernreisen verzichten und die Biologika-Therapie muss nicht verändert werden. Chickungunya-Infektionen spre-

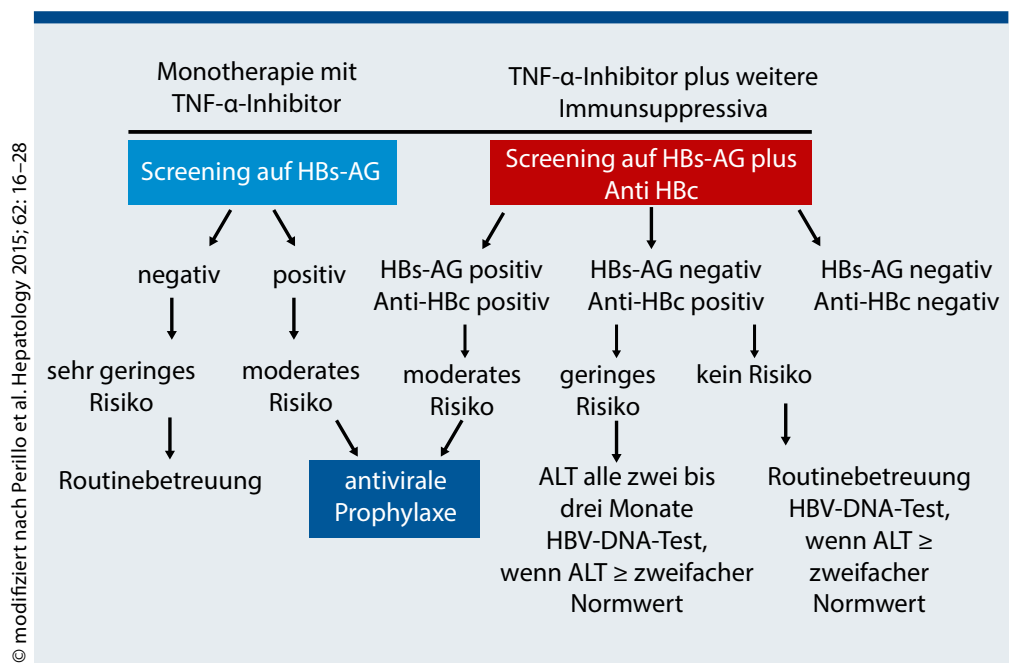

Abb. 1: Empfehlungen zum Hepatitis-B-Screening vor TNF-a-Blockade [modifiziert nach Perillo et al. Hepatology 2015; 62: 16-28] chen in der Regel gut auf NSAR an; gegebenenfalls kann die Therapie um niedrig-dosiertes Prednison ergänzt werden [6]. Bei einem kleinen Teil der Patienten kann es allerdings zu einem chronisch-destruierenden Verlauf kommen, der aber mit Methotrexat (MTX) beeinflussbar ist [7].

Ähnliches gilt für das Zika-Virus, ein weiteres Arbovirus, bei dem es nach der Infektion zu einer ähnlichen Symptomatik wie bei Chickungunya-Infektionen kommt, mit allerdings kürzerem Verlauf. Kneitz prophezeite, dass Arboviren Rheumatologen in der Zukunft vermehrt beschäftigen werden.

\section{Infektionsrisiko von Rheumapatienten}

Infektionen treten zu Beginn einer rheumatischen Erkrankung vermehrt auf. Im Verlauf zeigen sie eine signifikante Assoziation mit der Intensität der immunsuppressiven Therapie und bestimmen insbesondere bei Patienten mit Kollagenosen die Morbidität und Mortalität maßgeblich [8]. Bei Patienten mit Lupus erythematodes (LE) ist das Infektionsrisiko fünffach höher als bei solchen mit RA und wiederum doppelt so hoch, wenn eine Lupus-Nephritis vorliegt. Hydroxychloroquin scheint die Gefahr zu verringern.

Anders als zunächst erwartet, sind schwere Infektionen unter Rituximab (18,7 pro 100 Patientenjahre, PJ) nicht seltener als unter einer intravenösen Induktionstherapie mit Cyclophosphamid [9]. Als Risikofaktoren erwiesen sich eine Verminderung des IgG und der B-Zellen, eine Kreatinin-Clearance unter $45 \mathrm{ml} / \mathrm{min}$ sowie eine Prednison-Dosis von mehr als $15 \mathrm{mg} / \mathrm{d}$. Deutlich risikoreduzierend wirkte sich eine Pneumokokken-Impfung aus. Bei schweren Infektionen sollte die Indikation für eine IgG-Substitution geprüft werden.

\section{Infektionen unter Biologika}

Eine aktuelle Metaanalyse [10] hat die Einschätzung bestätigt, dass unter einer Therapie mit Biologika das Risiko schwerer Infektionen gegenüber DMARD erhöht ist (+ $30 \%$ ). Dies gilt insbesondere, wenn mit einer höheren als der empfohlenen Dosis behandelt wird. Für das Infektionsrisiko ist zudem entscheidend, ob die Patienten MTX-naiv sind oder nicht. MTX-Naive weisen ein geringeres Infektionsrisiko unter einer Biologika-Therapie auf als mit DMARD oder Biologika vorbehandelte Patienten. Daher sollte in der Zweitlinientherapie zwischen der optimalen Wirksamkeit eines Biologikums und möglichen Nebenwirkungen unter Berücksichtigung bestehender Komorbiditäten (zum Beispiel Diabetes mellitus) abgewogen werden. Wie Kneitz betonte, sind sicherlich nicht alle Biologika in dieser Hinsicht gleich zu bewerten. Die Datenlage sei aber für eine differenzierte Beurteilung bisher nicht ausreichend. 
Hier steht eine Anzeige.

算 Springer 
Bei Patienten, die eine schwere Infektion durchgemacht haben, ist das Risiko für eine erneute Infektion vier- bis fünffach erhöht. Dies müsse zwar bedacht werden, betonte Kneitz, allerdings sei es nicht zwangsläufig sinnvoll, deshalb auf einen TNF$\alpha$-Inhibitor zu verzichten. Denn das Infektionsrisiko in der Monotherapie sei unter konventionellen DMARD höher als unter der Monotherapie mit einem TNF- $\alpha$-Inhibitor.

\section{Biologika und Sepsis}

Wie eine aktuelle Auswertung der Daten des RABBIT-Registers ergab, ist das Sepsisrisiko unter einer TNF- $\alpha$-Inhibitor-Therapie gegenüber einer Therapie mit konventionellen DMARD erhöht, und zwar besonders bei älteren RA-Patienten und jenen mit Niereninsuffizienz [11]. Liegt eine Sepsis vor, vermindert sich unter der TNF- $\alpha$-Blockade hingegen das Risiko, an der Sepsis zu sterben. Die Inhibitoren tragen also dazu bei, dass eine schwere Infektion wie eine Sepsis oder Pneumonie milder verläuft. Dieses Phänomen wird als duale Rolle der TNF- $\alpha$-Inhibitoren bezeichnet: Sie erhöhen zwar das Infektionsrisiko, wirken aber bei manifester Infektion protektiv [12]. Gänzlich geklärt ist dieses Phänomen allerdings noch nicht.

\section{Infektionsrisiko unter Opioiden}

Was bisher häufig nicht bedacht werde, in der Praxis aber durchaus relevant sei, seien immunsuppressive Effekte von Opioiden, so Kneitz. Sie lassen die Zahl der Lymphozyten sinken und reduzieren die Aktivität der natürlichen Killerzellen. Diese in der Rheumatologie häufig verordneten Analgetika können daher ebenfalls das Infektionsrisiko erhöhen, wie eine große Fall-Kontroll-Studie mit fast 14.000 RA-Patienten ergab [13]. Die Infektinzidenz war in Phasen der Opioideinnahme um 40 \% erhöht. Dies galt auch für retardierte Opioide.

\section{Perioperatives Infektionsrisiko}

Das perioperative Infektionsrisiko bei rheumaorthopädischen Eingriffen wird als erhöht eingeschätzt. Dies gilt besonders für Infliximab, aber auch für klassische DMARD. Ob das Biologikum oder DMARD pausiert und ausgewaschen werden muss, sollte Kneitz zufolge anhand der individuellen Patientencharakteristika und in Abhängigkeit vom Eingriff entschieden werden. Ist die Wundfläche klein, keine Osteomyelitis vorhanden und das Gelenk nicht voroperiert, kann die BiologikaTherapie meist beibehalten werden. Andernfalls sei es sicherer, das Biologikum abzusetzen, auch wenn damit ein Schubrisiko verbunden ist. Infektionen infizierter Gelenkendoprothesen sind bei RA-Patienten deutlich häufiger als bei Arthrose-Patienten: 10,2 versus $5,7 \%$ [14].

\section{Screening auf Hepatitis B ist obligat}

Nicht nur auf eine Tuberkulose, sondern auch auf eine Hepatitis B muss vor Therapiebeginn mit Immunsuppressiva, insbesondere Biologika, gescreent werden, rief Kneitz ins Gedächtnis. Zu unterscheiden ist dabei zwischen einer aktiven (HBs-Antigen positiv), einer okkulten (Hbs-Ag negativ, anti-HBc positiv, anti-HBs negativ) und einer ausgeheilten Hepatitis B (Hbs-Ag negativ, anti-HBc und anti-HBs positiv). Diese differenzierte Betrachtung des Infektionsstatus ist für das weitere Vorgehen entscheidend. Denn das Reaktivierungsrisiko ist je nach geplanter Therapie unterschiedlich. Es ist unter Prednison ab $10 \mathrm{mg} / \mathrm{d}$ und unter Rituximab hoch, unter TNF- $\alpha$ Inhibitoren, Ustekinumab, Abatacept, niedrig dosierten oder maximal vier Wochen verabreichten Steroiden mittelhoch und unter Azathioprin, Methotrexat sowie intraartikulären oder maximal eine Woche oral applizierten Steroiden niedrig.

Zum weiteren Vorgehen gibt eine erste prospektive Studie bei 1.218 Patienten mit einer rheumatischen Erkrankung Auskunft, die den Einfluss auf eine vorbestehende Hepatitis B (anti-HBc pos, HBsAg neg) untersucht hat [15]. Sie kommt zu dem Ergebnis, dass eine Virusprophylaxe bei einer TNF- $\alpha$ Inhibitor-Monotherapie nicht pauschal erforderlich ist (Abb. 1). Ebenso wenig ist die routinemäßige Bestimmung der HBV-DNA geboten. Bei Kombination mit DMARD sollten allerdings regelmäßige Kontrollen erfolgen, da das Risiko einer Reaktivierung steigen könnte [16, 17].

Bei HBs-Ag-positiven, HBV-DNS-positiven Patienten ist die Indikation für eine Biologika-Therapie sehr kritisch zu hinterfragen. Die Einleitung einer antiviralen Therapie (Virustatika der zweiten oder dritten Generation) vor Beginn der Biologika-Therapie ist dann unumgänglich. Ist die Hepatitis B dagegen ausgeheilt, sollten nach einem Monat und im weiteren Verlauf alle drei bis sechs Monate Verlaufskontrollen durchgeführt werden [18]. Bei negativer Testung ist eine Impfung gegen Hepatitis B zu erwägen.

An einer Hepatitis C erkrankte Rheumapatienten profitieren von den heute verfügbaren Virustatika. In den meisten Fällen ist die Hepatitis $\mathrm{C}$ heilbar, sodass im Anschluss eine immunsuppressive Therapie begonnen werden kann. Persistiert die Hepatitis $\mathrm{C}$, muss ihre Aktivität regelmäßig kontrolliert werden. Bei Patienten mit einer akuten Hepatitis E riet Kneitz aufgrund der derzeitigen Datenlage dazu, die Immunsuppression auszusetzen.

Dr. Wiebke Kathmann

Rheuma-Update 2016, Wiesbaden, 26.-27.2.2016 


\section{Literatur}

1. Wormser et al. Arthritis Rheuma 2015; 67: 837-9

2. Sandberg MEC et al. Ann Rheuma Dis 2015; 74: 904-7

3. Weaver SC et al. N Engl J Med 2015; 372: 1231-9

4. Miner JJ et al. Arthritis Rheum 2015; 67: 1214-20

5. Brunier L et al. Joint Bone Spine 2015; Oct. 23 pii: S1297-319X

6. Rosario V et al. Can Rheumatol 2015; 34 : 1285-7

7. Javelle E et al. PLoS Negl Trop Dis 2015; 9: e0003603; doi: 10.1371

8. Feldman $\mathrm{CH}$ et al. Arthritis Rheum 2015; 67: 1577-85

9. Jones RB et al. N Engl J Med 2010; 363: 21120

10. Singh JA et al. Lancet $2015 ; 386: 258-65$

11. Richter A et al. Ann Rheum Dis 2015; doi:10.1136/annrheumdis-2015-207838

12. Strangfeld A et al. DGRH 2015, RA.09

13. Wiese $A$ et al. Arthritis Rheuma 2015; doi: 10.1002/art.39462

14. Singh et al. Arthritis Care Res 2015; 67: 71824

15. Barone M et al. Hepatology $2015 ; 62: 40-6$

16. Perillo et al. Hepatology 2015; 62: 16-28 und Gastroenterology 2015; 148: 221-44

17. Perillo et al. Gastroenterology 2015; 148 : 221-44

18. De Nard F et al. World J Hepatol 2015; 7: 344-61 